\title{
VARIACIONES EN LOS PATRONES DE PIGMENTACION Y DE COLOR DE LARVAS DE POBLACIONES NATURALES DE ANOPHELES ALBIMANUS DE COLOMBIA *
}

\author{
MARCO F. SUAREZ*, SUDHIR K. NARANG**, JACK A. SEAWRIGHT**
}

\begin{abstract}
Fueron observados el patrón de pigmentación y el color del cuerpo de las larvas de III y IV estadio en poblaciones naturales de Anopheles albimanus procedentes de cuatro áreas geográficas y ecológicas diferentes. Se incluyen tres variantes de la cápsula de la cabeza, el sable curvado de las antenas y la pigmentación; nueve variantes de la pigmentación del cuerpo y 17 del color del cuerpo. Se informa la alta variación en los patrones de color y otras características visibles. Se incluye una breve descripción de cada variante y la frecuencia observada en cada población.
\end{abstract}

\section{INTRODUCCION}

Los patrones de coloración de las larvas de muchas especies de mosquitos son altamente variables, incluídos los de Anopheles albimanus (1). La mayoría de tales variaciones son atribuidas a factores genéticos, cuya expresión puede variar con las condiciones fisiológicas y ambientales. Por esta razón, usualmente, se encuentran más variaciones en poblaciones naturales que en colonias de laboratorio. Las bases genéticas de muchos mutantes larvarios de An. albimanus han sido estudiadas siendo cada variante monofactorial en la herencia. Algunos ejemplos de los mutantes de larvas de $A n$. albimanus son: verde $(\mathrm{gl})$, ébano (eb), negro (bl), amarillo (y), Cafe (bw), sable ( $(\mathrm{sa})$, rayado $\underline{(\mathrm{st}+)}$, tórax blanco $\underline{(\mathrm{stw})}(2,3)$.

Los factores fisiológicos tales como la edad, afectan los patrones de pigmentación de los segmentos abdominales y de las antenas en las poblaciones de laboratorio de An. albimanus (4). Los factores ambientales tales como: la luz, la temperatura, los nutrientes también pueden influenciar los patrones de coloración.

Durante la determinación taxonómica de la especie de las larvas de los anofelinos recolectadas, hemos observado una alta variación en los patrones de color y otras características visibles. El propósito de la presente nota es presentar una breve descripción de cada tipo de variante en relación con los mutantes ya mapeados y su frecuencia, en cuatro poblaciones de diferentes áreas geográficas y ecológicas de Colombia.

\section{MATERIALES Y METODOS}

Las larvas fueron colectadas en tres lugares diferentes de la costa Atlántica -poblaciones 1 a 3- y, uno con la costa Pacífica -población 4- en Colombia. 1. Moñitos. En criaderos de agua salobre sobre la costa, localizados al norte de Montería en el departamento de Córdoba. 2. Padula. En criaderos de agua dulce a $25 \mathrm{Km}$ del Carmen de Bolívar. 3. Arjona. En pequeños pozos de agua de lluvias, localizados a $45 \mathrm{~km}$ al sur de Cartagena y a $3 \mathrm{~km}$ al norte del Canal del Dique sobre la carretera que conduce a Cartagena. 4. Gamboa. En un estanque artificial de agua dulce utilizado para el cultivo de camarones, abandonado, ubicado en la ciudad de Buenaventura en la costa Pacífica.

Las larvas de III y IV estadio fueron examinadas usando un estereoscopio. Se observaron las variaciones en el patrón de color y pigmentación de la cabeza,

\footnotetext{
$1 /$ Financiado en parte por U.S. National Academy of Sciences/National Research Council, Board on Science and Technology for International Development - BOSTID. CRG - Grant No. MVR-CO-2-85-46.

- Grupo de Entomología, Programa antimalárico. Servicio de Erradicación de la Malaria, Ministerio de Salud, Apartado Aéreo 4851, Bogotá, Colombia.

** ARS, USDA. Insects Affecting Man and Anımals Research Laboratory. P.O. Box 14565, Gainesville, FL 32604, U.S.A.
} 
antenas, tórax y abdomen. Algunos patrones de color y/o pigmentación encontrados fueron diferentes a los tipos descritos (3), y además se observaron patrones o coloraciones sobrepuestos. Cada larva fue asignada a una categoría y la frecuencia se expresó como un porcentaje, calculado a partir de los diversos tipos de patrones observados.

\section{RESULTADOS Y DISCUSION}

Los patrones y la pigmentación fueron altamente variables en An. albimanus. Algunas variantes fueron similares a los mutantes de los cuales se conoce la herencia, mientras otras variantes no han sido informadas antes. En las diferentes poblaciones fueron observadas grandes variaciones en las frecuencias de estas variantes (tablas 1, 2 y 3). A continuación se incluye una breve descripción de cada tipo.

\section{Coloración de la cápsula de la cabeza (Tabla 1)}

1. Cabeza acanelada. El color canela claro fue similar al del tipo más común observado en la colonia de laboratorio de An. albimanus del Instituto Nacional de Salud, en Bogotá a la del laboratorio Insects Affecting Man and Animals Research, USDA en Gainesville, Florida, Estados Unidos. En algunos casos no había pigmento en la cutícula de la cápsula de la cabeza.
2. Cabeza oscura. Fue muy similar al color de la cabeza del mutante ébano (eb) homocigoto descrito por Benedict et al. (5). Esta variante difiere de eb en que se presenta independiente del color del cuerpo. Se observaron larvas con cabeza oscura que tenían el cuerpo verde, café, canela claro, rosado o negro.

3. Cabeza negra clara. Las larvas tenían la cabeza de un color aparentemente intermedio entre los tipos 1 y 2 antes descritos. Esta coloración es muy similar al color de la cabeza de los heterocigotos ebano (5). En esta categoría están incluidas las variantes con el color de la cabeza que va de café a negro claro.

\section{Variaciones en la pigmentación del cuerpo (Tabla 2)}

1. Franja blanca. Similar al mutante rayado $\underline{(\mathrm{st}+)}$ publicado por Georghiou $(6)$ y mapeado en el brazo derecho del cromosoma $3(7,8)$; y sin franja, el cual fue el tipo más común de apariencia similar al mutante no rayado (st-) (2).

2. Franja roja. Similar al fenotipo franja blanca, excepto que el pigmento sobre el tórax y la línea media abdominal eran de color rojo. Adicionalmente, se observaron muchas larvas con una mezcla de pigmentos blancos y rojos.

\footnotetext{
Tabla 1

Tabla 1

$\sqrt{10}$

Variaciones en el color de la cápsula de la cabeza de las larvas de poblaciones naturales de Anopheles albimanus.
}

\begin{tabular}{lcccc} 
& & Número y porcentaje de las poblaciones \\
\cline { 3 - 5 } Variantes & Arjona & Padula & Moñitos & Gamboa \\
\hline Negra oscura & $69(31)$ & $5(10)$ & $2(4)$ & 0 \\
Carmelita & $54(24)$ & $15(30)$ & $32(64)$ & $16(16)$ \\
Negra clara & $99(45)$ & $30(60)$ & 50 & 106 \\
\hline Total & 222 & 50 & & \\
\hline
\end{tabular}


Tabla 2

Variaciones en la pigmentación del cuerpo de las larvas

de poblaciones naturales de Anopheles albimanus.

Número y porcentaje de poblaciones

\begin{tabular}{|c|c|c|c|c|}
\hline Variantes & Arjona & Padula & Moñitos & Gamboa \\
\hline \multicolumn{5}{|l|}{ Franja } \\
\hline Blanca & $28(9,7)$ & $4(7,3)$ & $2(4,3)$ & $21(19,1)$ \\
\hline Roja & $2(0,7)$ & $1(1,8)$ & 0 & $1(0,9)$ \\
\hline $\mathrm{Blanca}+\mathrm{roja}$ & $2(0,7)$ & $1(1,8)$ & $1(2,1)$ & $10(9,1)$ \\
\hline
\end{tabular}

\section{Sin franja}

Sin pigmento

o pigmento

al azar

Pigmento rojo

$176(60,9)$

$33(60,0)$

$35(74,5)$

$72(65,4)$

$\mathrm{Blanco}+$ rojo

$25(8,7)$

$2(3,6)$

$3(6,4)$

0

$12(4,2)$

$1(1,8)$

0

0

Torax

\begin{tabular}{lrrrr} 
Blanco & $36(12,5)$ & $13(23,6)$ & $6(12,8)$ & $5(4,5)$ \\
Rojo & $6(2,1)$ & 0 & 0 & $1(0,9)$ \\
Blanco + rojo & $2(0,7)$ & 0 & 0 & 0 \\
\hline tal & 289 & 55 & 47 & 110 \\
\hline
\end{tabular}

3. Tórax blanco. Similar al mutante de tórax blanco descrito por Kaiser et al. (9). La pigmentación blanca estaba restringida solamente al lado dorsal del tórax de las larvas y las pupas. Fueron observadas muchas variaciones en forma, color y localización de los pigmentos en el tórax. Las variaciones en la forma consistían de una configuración de balón de aire, muy similar a la fotografía publicada por Kaiser et al. (9); configuración triangular, en triángulo invertido, barril hueco similar al fenotipo de balón de aire, excepto por la pérdida de pigmentos blancos en la región media del tórax; configuración dividida donde los pigmentos blancos estaban concentrados sólo sobre el lado lateral del dorso del tórax con casi nada de pigmentos sobre la parte media del tórax, y menos frecuente en la forma de herradura y la forma rectangular. La variación en el color del pigmento sobre el tórax consiste en tórax rojo y una mezcla de pigmento rojo y blanco.

\section{Patrones de pigmentación sobre los segmentos abdominales}

Prescindiendo de si la larva era sin franja, con franja, tórax blanco o tórax rojo, se encontraron patrones adicionales de pigmentación sobre unos pocos segmentos abdominales. Una parte de la variación fue 
observada con respecto al número de segmentos implicados y a la intensidad diferencial de pigmentación sobre estos segmentos. El patrón más común consistió de dos bandas, una sobre cada lado de la línea media en los segmentos 2 y 5 y una banda sobre el segmento 8 conformando un patrón 2-5-8. El color de los pigmentos fue blanco, rojo o una mezcla de ambos sobre los segmentos 2 y 5 , pero el segmento 8 casi siempre tenía pigmentos blancos. Fueron tantas las variaciones en los patrones compuestos, a causa de los diferentes segmentos implicados en cada tipo, que pudo observarse una gran combinación de patrones.
Variación en el color del cuerpo (Tabla 3)

1. Verde. Fue muy similar al mutante verde $(\mathrm{gl})$ descrito y mapeado en el brazo derecho del cromosoma 2 (10).

2. Café. Fue muy similar al mutante café (bw) mapeado en el brazo izquierdo del cromosoma 2 (1).

3. Canela o café claro. Fue similar al canela o café claro generalmente referido como el tipo silvestre (1).

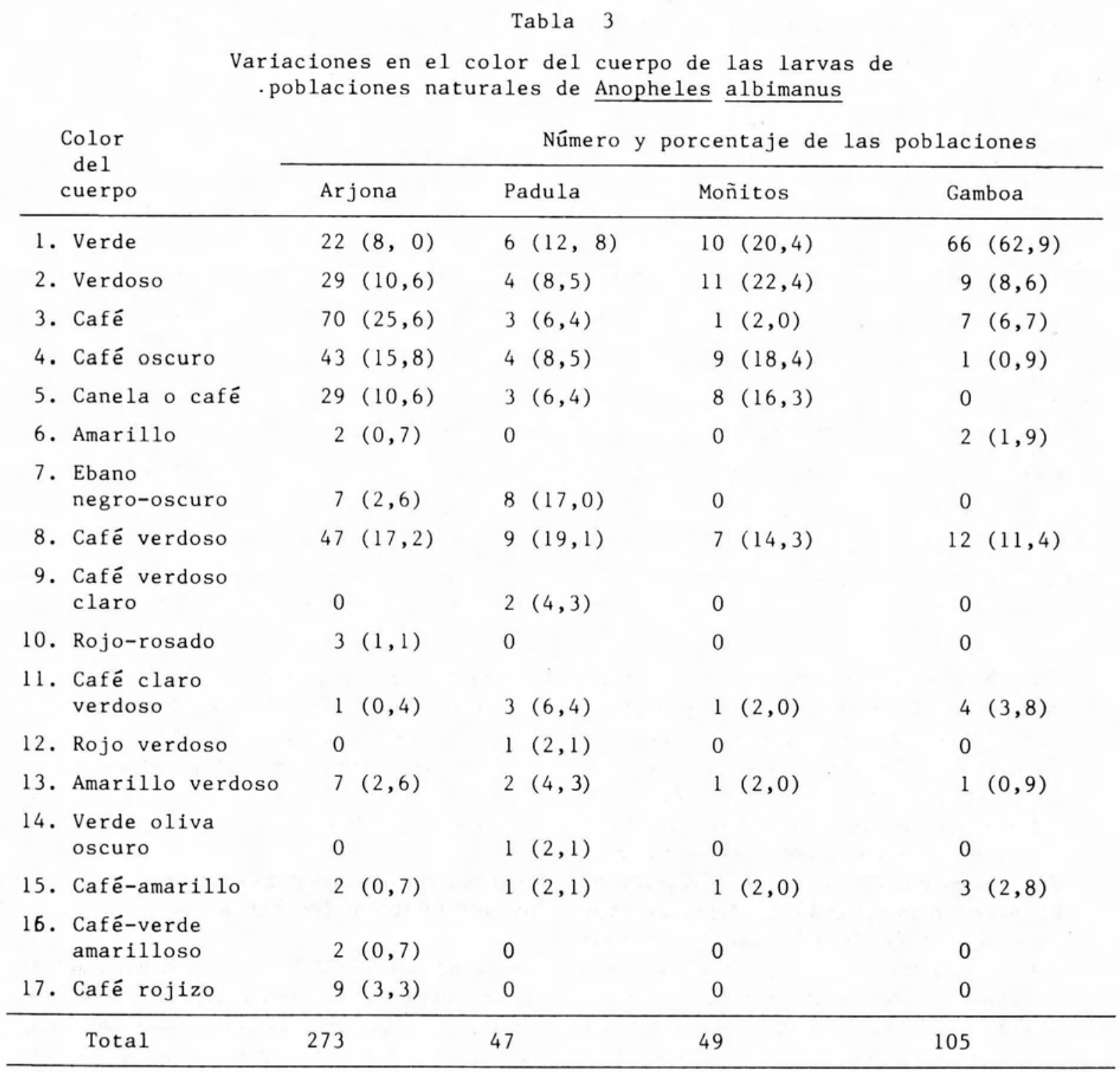


4. Amarillo. Fue muy similar al mutante amarillo $(\mathrm{y})$, mapeado en el brazo derecho del cromosoma 2 (1).

5. Negro. Fue similar al color del cuerpo observado en el mutante (eb) (5), excepto que a diferencia de eb el color de la cápsula de la cabeza fue independiente del color del cuerpo.

Adicionalmente a los 5 tipos mencionados, se observaron muchas variantes con colores intermedios o combinaciones de varios colores tales como: café verdoso (similar al mutante descrito en An. stephensi por Sharma) (11), café rosado, café amarillento, rojo claro, rojo verdoso, amarillo verdoso y verde oliva oscuro.

\section{Variación de las antenas}

1. Sable antenal curvado. Solamente en una muestra $(n=287)$ de la población de Arjona, los sables antenales ventral y dorsal de la antena izquierda eran curvados hacia adentro cerca a una porción perpendicular al eje principal.

2. Variaciones de pigmentación. Observamos una alta variación en la intensidad y distribución de pigmentos negros sobre el eje de la antena. Algunas larvas tenían antenas canela transparentes o claras con nada o casi nada de pigmentos. Por otra parte, en algunos, el eje de la antena era oscuro. La mayoría de las antenas de las larvas eran de tipos intermedios diferenciándose en el tamaño de los segmentos oscuro y claro del eje. Estas variaciones se sobreponen considerablemente.

Los hallazgos muestran la existencia de variabilidad fenotípica en las poblaciones naturales de las larvas de An. albimanus. Las variaciones en el color del cuerpo o en los patrones de pigmentación observados en estas poblaciones de campo, pueden ser una representación de una combinación de factores genéticos y/o ambientales. Esta variabilidad fenotípica podría representar la existencia de variabilidad genética lo que podría relacionarse con otras características fenotípicas, como la capacidad vectorial; sin embargo, es necesario realizar estudios para confirmarlo.

\section{SUMMARY}

Color and pigmentation variants of Anopheles albimanus of third and fourth instar larvae were described from field populations in four different geographic and ecological areas in Colombia, together with their frecuency. These variants include three of the head capsule, nine of body pigmentation and of body color. Also, curved antennal sabers and antennal pigmentation are described. We report the ocurrence of high level of polymorphys in body color, pigmentation pattern and other visible traits. A brief description of each variant its frequency in each populations is included.

\section{AGRADECIMIENTOS}

Los autores expresan su agradecimiento a los doctores José S. Forero y Alejandro Rodríguez, por su continuo apoyo, al doctor Antonio Donado por la colaboración en el apoyo logístico y a los señores Guillermo Berruecos, Rafael Lafont y Lalo Omar García, de las zonas SEM de Barranquilla, Montería y Buenaventura respectivamente, por la ayuda en la recolección de las larvas.

\section{BIBLIOGRAFIA}

1. Seawright JA, Benedict MQ, and Narang SK.: Color mutants in Anopheles albimanus (Diptera Culicidae). Ann. Entomol Soc Am 1985; 78:177.

2. Narang S, Seawright JA.: Linkage relationships and genetic mapping in Culex and Anopheles. In "Recent developments in the genetics of insect disease vectors" (Steiner et al. eds) 1982; Stipes Publishing Co., Champaing, I11. pp. 231-289.

3. Narang SK, Seawright JA.: Linkage map of Anopheles albimanus. In "Linkage maps" (S. O'Brien, ed.) 1986; Cold Spring Harbor Laboratory (En prensa).

4. Childress L, Vernon Jr.: The genetics of three morphological mutant markers of the mosquito Anopheles albimanus Wiedemann. M.S. Thesis, University of Florida, 1977; pp. 50.

5. Benedict MQ, Seawrigth JA, Anthony DW, and Avery SW.: Ebony, a semidominant lethal mutant in the mosquito Anopheles albimanus. Can J Genet Cytol 1979; 21:193.

6. Georghiou GP, Gidden FE, and Cameron JW.: A stripe character in Anopheles albimanus and its linkage relationships 
to sex and dieldrin resistance. Ann. Entomol Soc Amer 1967; 60:323.

7. Rabbani MG, Seawrigth JA.: Use of Y-autosome translocations in assigning the stripe locus to chromosome 3 in the mosquito Anopheles albimanus. Ann. Entomol Soc Am 1976; 69:266.

8. Seawrigth JA, Benedict MQ, and Narang S.: Use of deficiencies for mapping four mutart loci on the salivary gland chromosomes of Anopheles albimanus. Theor Appl Genet 1983; 65:207.
9. Kaiser PE, Benedict MQ, and Seawrigth JA.: A White thorax mutant and its relationship in an allelic series in Anopheles albimanus Wiedernann. Mosq. News 1981; 41:455.

10. Seawrigth JA, Childres LV, and Benedict MQ.: Genetics of green larva, a recessive mutant on chromosome 2 in Anopheles albimanus Wiedemann. Mosq. News 1981; 41:455.

11. Sharma VP, Subbarao SK, Ansari MA, and Razdan RK.: Inheritance pattern of two new mutants, red eye and greenishbrown larva in Anopheles stephensi. Mosq. News 1979; 39:655. 